\title{
Reconstruction of Volumetric Reflectance using Spatio-Sequential Frequency Correlation Imaging
}

\author{
Tsuyoshi Takatani \\ Nara Institute of Science and \\ Technology (NAIST)
}

\author{
Takahito Aoto \\ University of Tsukuba
}

\author{
Kenichiro Tanaka \\ Nara Institute of Science and \\ Technology (NAIST)
}

\author{
Takuya Funatomi \\ Nara Institute of Science and \\ Technology (NAIST)
}

\author{
Yasuhiro Mukaigawa \\ Nara Institute of Science and \\ Technology (NAIST)
}

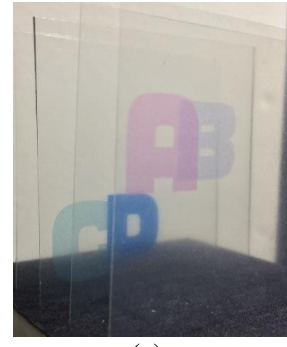

(a)

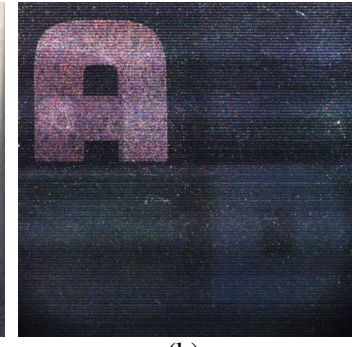

(b)

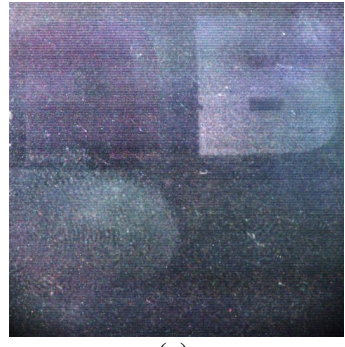

(c)

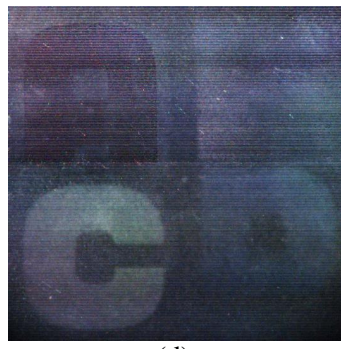

(d)

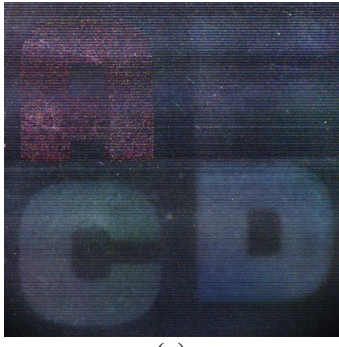

(e)

Figure 1: Reconstruction results from real data. (a) Test object consisting of four overhead projector sheets spaced with gaps of $10 \mathrm{~mm}$. (b-e) Reconstructed volumetric reflectance at each of the four sheets.

\begin{abstract}
In this paper, we propose a novel pro-cam technique for reconstruction of the volumetric reflectance inside an object. The key concept is use of spatio-sequentially modulated illumination to extract only the required signal at a 3D point. We discovered an effect where a projector and camera pair with different focal lengths naturally produced a spatial frequency modulation. By combining this effect with a direct conversion technique to demodulate the signals, the resulting spatio-sequential frequency correlation enables reconstruction of the reflectance at a $3 \mathrm{D}$ point within the object. Experimental results based on both synthetic and real data show that the proposed method can reconstruct volumetric reflectance discretely.
\end{abstract}

\section{CCS CONCEPTS}

- Computing methodologies $\rightarrow$ Computational photography; Reconstruction;

\section{KEYWORDS}

direct conversion, pro-cam system, correlation imaging

Permission to make digital or hard copies of all or part of this work for personal or classroom use is granted without fee provided that copies are not made or distributed for profit or commercial advantage and that copies bear this notice and the full citation on the first page. Copyrights for components of this work owned by others than ACM must be honored. Abstracting with credit is permitted. To copy otherwise, or republish, to post on servers or to redistribute to lists, requires prior specific permission and/or a fee. Request permissions from permissions@acm.org.

SA '18 Technical Briefs, December 4-7, 2018, Tokyo, Japan

(C) 2018 Association for Computing Machinery.

ACM ISBN 978-1-4503-6062-3/18/12 . \$15.00

https://doi.org/10.1145/3283254.3283278

\section{ACM Reference Format:}

Tsuyoshi Takatani, Takahito Aoto, Kenichiro Tanaka, Takuya Funatomi, and Yasuhiro Mukaigawa. 2018. Reconstruction of Volumetric Reflectance using Spatio-Sequential Frequency Correlation Imaging. In SIGGRAPH Asia 2018 Technical Briefs (SA '18 Technical Briefs ), December 4-7, 2018, Tokyo, Japan. ACM, New York, NY, USA, 4 pages. https://doi.org/10.1145/3283254. 3283278

\section{INTRODUCTION}

Observation of the interior of an object is a difficult task but is important in a wide variety of applications, including biological imaging, medical imaging, and industrial inspection. Various imaging techniques have therefore been developed to achieve clear observation inside objects, including X-ray fluorescence technique [Dik et al. 2008] and infrared reflectography [Gavrilov et al. 2008]. These techniques use different features of electromagnetic waves but require expensive equipment that is not readily available. Therefore, we have focused on use of a projector-camera (pro-cam) system, which is readily available at low cost.

In this paper, we propose a novel pro-cam technique for volumetric reflectance reconstruction inside a target object. The proposed technique requires a projector and camera pair with different focal lengths, which is a common situation for most pro-cam systems. We discovered the difference between their focal lengths causes a natural spatial frequency modulation called depth-dependent frequency. In addition, by combining this modulation with direct conversion, a well-known signal processing technique, a spatio-sequential frequency correlation leads to reconstruction of the reflectance of a 3D point within the object. Experiments are performed using both synthetic and real data to evaluate the proposed method. 


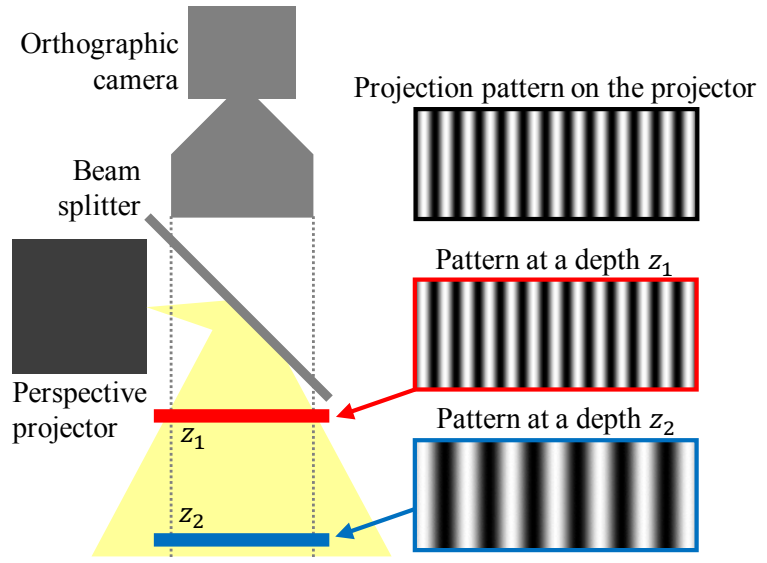

Figure 2: Illustration of pro-cam system composed of a perspective projector and an orthographic camera.

Pro-cam systems have often been used for 3D shape reconstruction [Geng 2011] but are also useful for imaging of the interior of a target object. Nayar et al. [2006] proposed the high-frequency illumination (HFI) to separate observations into two components that were based on direct and global illuminations. The global illumination includes important signals from the interior of the object. Fuchs et al. [2008] used confocal imaging to recover an image of a solid target in a scattering medium. Achar and Narasimhan [2014] extended HFI for a multi-focus projector to allow simultaneous recovery of the object shape and the global illumination. In this method, the projector focus is varied mechanically to provide spatiotemporal modulation; while this is similar to our idea, the method proposed in this paper does not require the focus to be changed. Tanaka et al. [2017] extended HFI to multi-frequency illumination to recover slices of the interior of a translucent object. This approach is also similar to our proposed method but it requires an empirical optimization because it is not based on a modulation technique.

Many pro-cam systems have been described in the literature but the depth-dependent frequency that we discovered has not been reported previously, while its use in combination with direct conversion for spatio-sequential frequency correlation is also novel.

\section{FREQUENCY CORRELATION IMAGING}

We introduce a pro-cam system composed of a perspective projector and an orthographic camera, as shown in Fig. 2, where both devices are collocated. Note that this is a simple example for explanation of the concept and, in general, a projector and camera pair with different focal lengths is required. When projecting a sinusoidal pattern with frequency $f_{1}$ at depth $z_{1}(>0)$ and placing a white board at the depth $z_{1}$, the frequency that is observed by the camera is also $f_{1}$. Next, when the board is moved to a different depth $z_{2}\left(>z_{1}\right)$, which is further than $z_{1}$ from the system, the observed frequency $f_{2}$ is lower than $f_{1}$. This occurs because the orthographic camera captures a scene that is always the same size, regardless of the distance, while a projected pattern from the perspective projector broadens spatially with increasing distance. We use this natural frequency modulation, which is called depth-dependent frequency, for correlation imaging.

\subsection{Observation model}

For simplicity, assuming that a 1D sinusoidal pattern is projected along an axis, e.g. $x$, the illumination at a $3 \mathrm{D}$ point $(x, y, z)$ is written as;

$$
L(x, y, z)=D(z)+A(z) \sin (\omega(z) x+\phi),
$$

where $D(\cdot)$ is the offset, $A(\cdot)$ is the amplitude, $\omega(\cdot)$ is the angular frequency, and $\phi$ is the phase of the sinusoidal pattern that is projected to the point. $(x, y)$ represents the position on the projection/image planes and $z$ is the depth from these planes. As described above, the frequency $\omega(z)$ varies depending on the depth $z$. When the frequency $\omega_{1}$ at a depth $z_{1}$ is determined, it can then be formulated as;

$$
\omega(z)=\frac{z_{1}}{z} \omega_{1} .
$$

This is the depth-dependent frequency, which is modulated naturally. Note that the phase does not vary for different depths.

The observed intensity of a pixel $(x, y)$ on the image plane is represented using an integral of all the intensities at the different depths, as;

$$
I(x, y)=\int_{z \geq z_{1}} I_{p}(x, y, z) d z,
$$

where $I_{p}(\cdot)$ is the intensity at the point $(x, y, z)$. We assume that no objects are present between the system and the depth $z_{1}$. When the point, which has the reflectance of $R(x, y, z)$, is illuminated by $L(x, y, z)$, the intensity can be written as;

$$
I_{p}(x, y, z)=L(x, y, z) R(x, y, z) E(x, y, z),
$$

where $E(\cdot)$ is the attenuation rate at which reflected light from the point reaches the pixel. Let the transmittance of the point be $T(x, y, z)$; the attenuation rate can then be formulated as;

$$
E(x, y, z)=\prod_{z_{1}}^{z} T(x, y, \zeta)^{d \zeta} .
$$

Ideally, the offset $D(z)$ and the amplitude $A(z)$ of the projected pattern are homogeneous at a depth $z$ but both are affected by the scene. For example, illumination to a depth $z_{2}\left(>z_{1}\right)$ may be attenuated by points at the depth $z_{1}$, which results in an inhomogeneous offset $D(x, y, z)$ and amplitude $A(x, y, z)$. When the offset $D\left(z_{1}\right)$ and the amplitude $A\left(z_{1}\right)$ at the depth $z_{1}$ have been determined, the offset and the amplitude of a projected pattern to a point $(x, y, z)$ can be written as;

$$
\begin{aligned}
& D(x, y, z)=D\left(z_{0}\right) F(x, y, z), \\
& A(x, y, z)=A\left(z_{0}\right) F(x, y, z), \\
& F(x, y, z)=\prod_{z_{1}}^{z} T(\hat{x}, \hat{y}, \zeta)^{d \zeta},
\end{aligned}
$$

where $\hat{x}=\frac{x \zeta}{z}$ and $\hat{y}=\frac{y \zeta}{z} . F(\cdot)$ denotes the attenuation rate of a light ray from depth $z_{1}$ to a point $(x, y, z)$.

Finally, the observed intensity of a pixel $(x, y)$ is expressed as;

$$
I(x, y)=\int_{z \geq z_{1}}\left\{D^{*}(x, y, z)+A^{*}(x, y, z) \sin (\omega(z) x+\phi)\right\} d z
$$

where

$$
\begin{aligned}
& D^{*}(x, y, z)=D(x, y, z) R(x, y, z) E(x, y, z), \\
& A^{*}(x, y, z)=A(x, y, z) R(x, y, z) E(x, y, z) .
\end{aligned}
$$




\subsection{Reconstruction using direct conversion}

The problem to be solved is the reconstruction of the reflectance $R(x, y, z)$ for all points. The observed intensity is spatially modulated by the depth-dependent frequency; therefore, spatial correlation could lead to extraction of a signal with a specific frequency. However, the spatial correlation is affected by the spatial frequency of the reflectance map. To resolve this problem, we apply direct conversion, which was invented by Colebrook [1924] and can detect a signal with a specific frequency within complex signals. To "temporally" modulate the signals, we change the frequency of a sinusoidal pattern sequentially multiple times. The observed in tensity of the pixel $(x, y)$ when the depth-dependent frequency is $\omega(z)[i]$ is written as;

$$
I(x, y)[i]=\int_{z \geq z_{1}}\left\{D^{*}(x, y, z)+A^{*}(x, y, z) \sin (\omega(z)[i] x+\phi)\right\} d z,
$$

where $i$ indicates a sequential index. Next, use of a sinusoidal signal with a frequency $\omega(w)[i]$ to multiply both sides of Eq. (12) gives

$$
\begin{aligned}
& I(x, y)[i] \sin (\omega(w)[i] x) \\
& =\int_{z \geq z_{1}}\left\{D^{*}(x, y, z) \sin (\omega(w)[i] x)\right. \\
& \quad-\frac{A^{*}(x, y, z)}{2} \cos ((\omega(z)[i]+\omega(w)[i]) x+\phi) \\
& \left.\quad+\frac{A^{*}(x, y, z)}{2} \cos ((\omega(z)[i]-\omega(w)[i]) x+\phi)\right\} d z .
\end{aligned}
$$

All terms in Eq. (13) are now dependent on the sequential index $i$. In the case where $z=w, \omega(z)[i]-\omega(w)[i]=0$ and the third term then becomes independent of the sequential index, i.e., the third term alone becomes a DC component of the sequential signals $I(x, y)[i] \sin (\omega(w)[i] x)$. The DC component can then be extracted easily using a discrete Fourier transform (DFT). When this operator is defined as $\langle\mathcal{F}\{\cdot\}\rangle_{\mathrm{DC}}$, the third term in Eq. (13) can then be extracted as;

$$
\langle\mathcal{F}\{I(x, y)[i] \sin (\omega(w)[i] x)\}\rangle_{\mathrm{DC}}=\frac{A^{*}(x, y, w)}{2} \cos \phi .
$$

Additionally, multiplying both sides of Eq. (12) by $\cos (\omega(w)[i] x)$ gives

$$
\langle\mathcal{F}\{I(x, y)[i] \cos (\omega(w)[i] x)\}\rangle_{\mathrm{DC}}=\frac{A^{*}(x, y, w)}{2} \sin \phi .
$$

Because $\cos ^{2} \phi+\sin ^{2} \phi=1$, we can obtain $A^{*}(x, y, w)$ for any point $(x, y, w)$ using the computation shown below;

$$
\begin{aligned}
A^{*}(x, y, w)=2\left\{\langle\mathcal{F}\{I(x, y)[i] \sin (\omega(w)[i] x)\}\rangle_{\mathrm{DC}}^{2}\right. \\
\left.+\langle\mathcal{F}\{I(x, y)[i] \cos (\omega(w)[i] x)\}\rangle_{\mathrm{DC}}^{2}\right\}^{\frac{1}{2}} .
\end{aligned}
$$

Reconstruction of the reflectance begins at depth $z_{1}$ and then moves further from the system discretely. Based on Eq. (11), the reflectance at depth $z$ can generally be reconstructed as;

$$
R(x, y, z)=\frac{A^{*}(x, y, z)}{A(x, y, z) E(x, y, z)} .
$$

Assuming that there are no objects before the depth $z_{1}$, which means that $E\left(x, y, z_{1}\right)=1$, and the amplitude at the depth $z_{1}$, denoted by

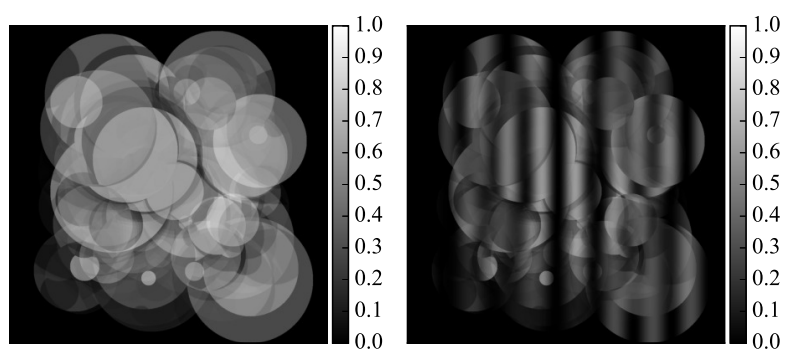

Figure 3: Rendered images of ten-layered object. The object is illuminated on the left using a completely white pattern, while a sinusoidal pattern is used on the right. The bars on the right of the images indicate the reflectance.

$A\left(z_{1}\right)$, is known, Eq. (17) at the depth $z_{1}$ then becomes

$$
R\left(x, y, z_{1}\right)=\frac{A^{*}\left(x, y, z_{1}\right)}{A\left(z_{1}\right)} .
$$

Next, assuming that the transmittance is

$$
T(x, y, z)=1-R(x, y, z)
$$

the attenuation rate $E\left(x, y, z_{2}\right)$ and the amplitude $A\left(x, y, z_{2}\right)$ can be computed at a depth $z_{2}\left(=z_{1}+\delta z\right)$ using Eqs. (5) and (7), respectively. Therefore, the reflectance $R\left(x, y, z_{2}\right)$ can also be reconstructed using Eq. (17). Repeating this process in the same manner enables reconstruction of the reflectance of the scene.

\section{EXPERIMENTAL RESULTS}

\subsection{Synthetic data}

First, We evaluate the proposed method using synthetic data. In the simulation, the depth $z_{1}$ is set at $100 \mathrm{~mm}$, while the resolution of both the observed image and the projected pattern is set at $1000 \times 1000$ pixels; the offset and the amplitude at the depth $z_{1}$ are set at $D\left(z_{1}\right)=0.5$ and $A\left(z_{1}\right)=0.5$, respectively, because the maximum brightness of the projector is 1 . The frequency of a sinusoidal pattern at depth $z_{1}$ is set at every $0.001 \mathrm{~Hz}$ in the $0.001 \leq f\left(z_{1}\right)<0.5 \mathrm{~Hz}$ range, where the angular frequency $\omega(z)=2 \pi f(z)$. The target object consists of ten layers with a gap size of $10 \mathrm{~mm}$. Multiple circles with different reflectances are rendered on each of these layers, as shown in Fig. 3. Experimental results are shown in Fig. 4, which include the reference and reconstructed results for each of the layers with associated error maps. The peak signal-to-noise ratios (PSNRs) for the 1st and 2nd layers are 28.89 and $22.91 \mathrm{~dB}$, respectively. The reconstructed reflectances look good in these cases. The PSNRs for the 3rd to 10th layers are also given in the caption for Fig. 4. These results obviously show that it is more difficult to reconstruct the reflectances of the deeper layers. As the results show, the reconstruction errors around the central region are relatively large. This is because the light rays around the origin of the projection plane do not broaden sufficiently for the orthographic camera, which results in a depth-independent frequency.

\subsection{Real data}

We also evaluated the proposed method using real data. The experimental setup consisted of a laser projector (SK Telecom LB-UH6CB, $1280 \times 720)$ and a camera (FLIR Grasshopper3, $1920 \times 1440)$ with a 


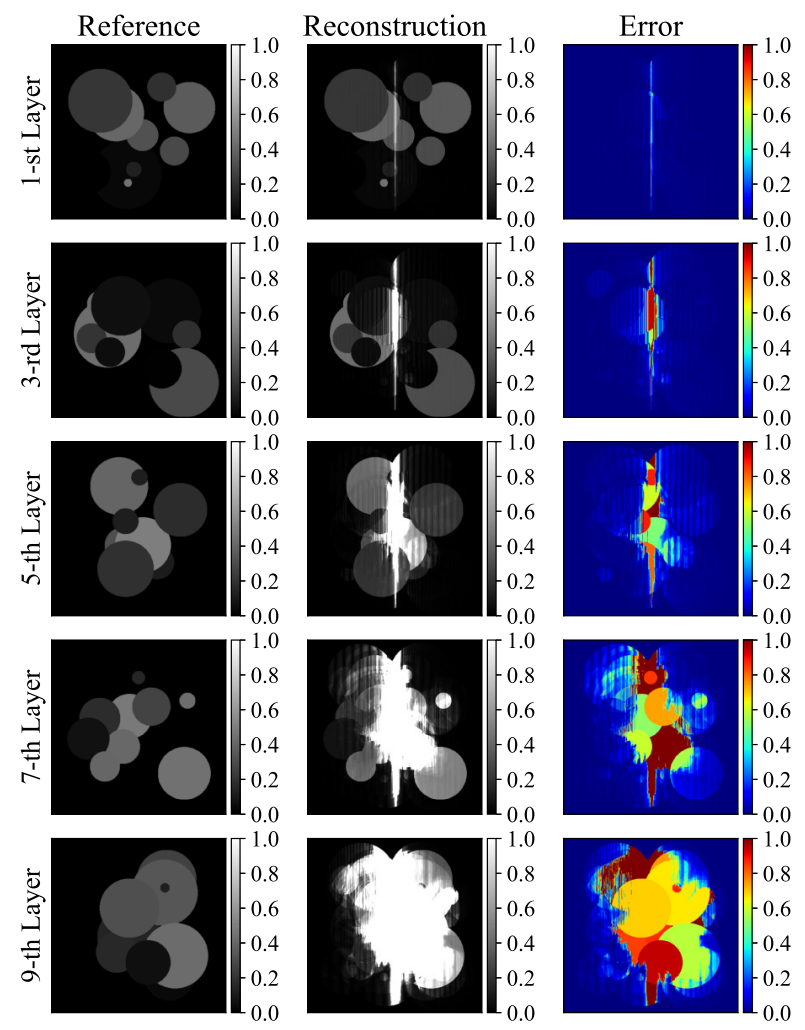

Figure 4: Evaluation of reconstructed reflectances on five of the ten layers. The peak signal-to-noise ratios from the 1st to 10th layers are $28.89,22.91,17.53,15.53,14.70,11.97,9.17$, $7.71,6.83$, and $4.95 \mathrm{~dB}$, respectively.

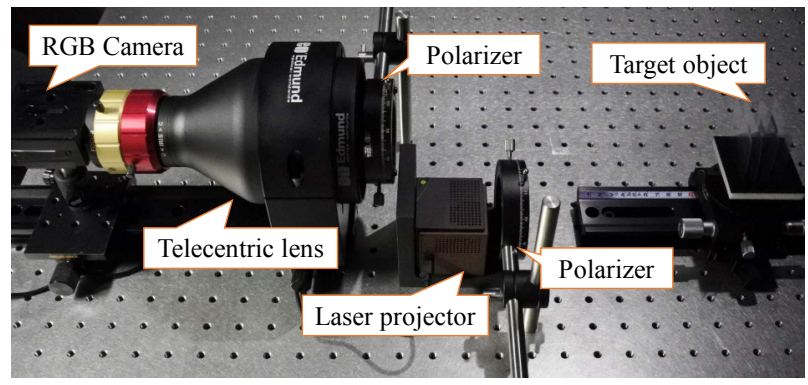

Figure 5: Experimental setup.

telecentric lens (Edmund Optics \#55-348), as shown in Fig. 5. Note here that we used linear polarizers to remove the specular reflection, which can make it difficult to observe the projected patterns. The offset and the amplitude at the depth $z_{1}(=252.83 \mathrm{~mm})$ are set at $D\left(z_{1}\right)=A\left(z_{1}\right)=127$. The frequency at the depth $z_{1}$ is set at every $0.78125 \mathrm{mHz}$ in the $0.78125 \leq f\left(z_{1}\right)<500 \mathrm{mHz}$ range. In practice, the laser projector is not perfectly focus-free and it has a working distance range. Our measurements indicate that the projector requires a working distance of at least $250 \mathrm{~mm}$ as to focus. The telecentric lens also has the practical limitations of a shallow depth of field (DoF) and a limited working distance. In the setup, the DoF is $60 \mathrm{~mm}$ and the closest working distance is $130 \mathrm{~mm}$.
The target consists of four overhead projector (OHP) sheets on which the letters A, B, C, and D have been printed. The gap between these sheets is set at $10 \mathrm{~mm}$. The reconstruction results are shown in Fig. 1. The A in Fig. 1(b) is quite clear when compared with the other letters. The B and C in (c) and (d), respectively, are clearer relative to the other letters. In (e), while both the $\mathrm{D}$ and the $\mathrm{C}$ can be seen, the A and B are almost invisible. The experimental results showed the proposed method can reconstruct the various reflectances inside the object.

\section{CONCLUSIONS}

We have proposed a novel pro-cam technique to reconstruct the volumetric reflectance inside a target object. The key concept is the spatio-sequentially modulated illumination that occurs in a pro-cam system in which the focal lengths of the projector and the camera are different. Combination of this illumination with direct conversion leads to reconstruction of the reflectance of a 3D point. The experimental results from both synthetic and real data showed that the proposed method discretely reconstructed the volumetric reflectance characteristics inside the objects.

In this case, the experimental results showed reconstruction of the volumetric reflectance in discrete layers. Reducing the gap can provide more detailed volumetric reflectance results but the depth resolution is limited by the sensitivity of direct conversion. Enhancing the modulation method could improve the depth resolution and this will form part of our future work. Additionally, we assume that the transmittance is as Eq. (19), which means that no absorption or scattering occurs inside the object. Because this makes the proposed method very limited, consideration of more realistic optical phenomena will be an important step in our future work.

\section{ACKNOWLEDGMENTS}

This work is partly supported by JSPS KAKEN grants JP17J05602, JP17H04686, JP18K19822, and 17K19979 and MIC/SCOPE \#181607013. We thank David MacDonald, MSc, from Edanz Group for editing a draft of this manuscript.

\section{REFERENCES}

Supreeth Achar and Srinivasa G Narasimhan. 2014. Multi focus structured light for recovering scene shape and global illumination. In European Conference on Computer Vision. Springer, 205-219.

F. M. Colebrook. 1924. Homodyne. Wireless World and Radio Rev. 13 (1924), 645-648. Joris Dik, Koen Janssens, Geert Van Der Snickt, Luuk van der Loeff, Karen Rickers, and Marine Cotte. 2008. Visualization of a lost painting by Vincent van Gogh using synchrotron radiation based X-ray fluorescence elemental mapping. Analytical chemistry 80, 16 (2008), 6436-6442.

Christian Fuchs, Michael Heinz, Marc Levoy, Hans-Peter Seidel, and Hendrik Lensch. 2008. Combining confocal imaging and descattering. In Computer Graphics Forum, Vol. 27. Wiley Online Library, 1245-1253.

D Gavrilov, C Ibarra-Castanedo, E Maeva, O Grube, X Maldague, and R Maev. 2008. Infrared methods in noninvasive inspection of artwork. In Proceedings of 9th international conference on NDT of art. 25-30.

Jason Geng. 2011. Structured-light 3D surface imaging: a tutorial. Advances in Optics and Photonics 3, 2 (2011), 128-160.

Shree K Nayar, Gurunandan Krishnan, Michael D Grossberg, and Ramesh Raskar. 2006. Fast separation of direct and global components of a scene using high frequency illumination. In ACM Transactions on Graphics (TOG), Vol. 25. ACM, 935-944.

Kenichiro Tanaka, Yasuhiro Mukaigawa, Hiroyuki Kubo, Yasuyuki Matsushita, and Yasushi Yagi. 2017. Recovering Inner Slices of Layered Translucent Objects by Multi-Frequency Illumination. IEEE transactions on pattern analysis and machine intelligence 39, 4 (2017), 746-757. 